PROCEEDINGS OF THE

AMERICAN MATHEMATICAL SOCIETY

Volume 131, Number 7, Pages 2241-2245

S 0002-9939(03)06432-3

Article electronically published on January 28, 2003

\title{
EVERY THREE-POINT SET IS ZERO DIMENSIONAL
}

\author{
DAVID L. FEARNLEY, L. FEARNLEY, AND J. W. LAMOREAUX \\ (Communicated by Alan Dow)
}

\begin{abstract}
This paper answers a question of Jan J. Dijkstra by giving a proof that all three-point sets are zero dimensional. It is known that all two-point sets are zero dimensional, and it is known that for all $n>3$, there are $n$-point sets which are not zero dimensional, so this paper answers the question for the last remaining case.
\end{abstract}

\section{INTRODUCTION}

An $n$-point set is a subset of the plane which intersects every straight line in the plane in exactly $n$ points. It is known that $n$-point sets exist for every $n$, and John Kulesza [4] has shown that that all two-point sets are zero dimensional, answering a question of R. D. Mauldin [5]. Khalid Bouhjar, Jan J. Dijkstra, Jan Van Mill [2] have shown that three-point sets cannot contain arcs, and that four-point sets and higher can contain arcs. It was asked by Dijkstra [1] whether three-point sets need always be zero dimensional. This is the question we address in this paper.

All the usual definitions of dimension coincide for separable metric spaces, as discussed in Engelking [3]. We will use small inductive dimension in this paper.

\section{ProOF OF THE THEOREM}

We will use the fact that no three-point set contains an arc in the process of proving this theorem. For completeness we also include a proof of that result after the proof of the main theorem.

Theorem 2.1. Every three-point set is zero dimensional.

Proof. Let $S$ be a three-point set and suppose by way of contradiction that $S$ is not zero dimensional. Then let $p$ be a point of $S$ and let $O$ be an open set in the plane containing $p$ such that no open set containing $p$ is contained in $O$ and has a boundary which is disjoint from $S$.

Let $q$ be a point of $S$ other than $p$, and refer to the direction from $p$ to $q$ as "up". We will define "left" as the direction to the left of a point moving from $p$ to $q$. Choose horizontal line segments above and below $p$ which are contained in $O$ and are below $q$ which do not contain points of $S$ directly above or below $p$. This is possible because there are only three points of $S$ on the vertical line through $p$. Since there are at most three points on each such horizontal line segment which are contained

Received by the editors September 7, 2000 and, in revised form, April 27, 2001.

2000 Mathematics Subject Classification. Primary 54B05, 54H05, 54F45.

Key words and phrases. $n$-point set, zero dimensonal.

(C)2003 American Mathematical Society 
in $S$, there are intervals contained in these horizontal segments, directly above and below $p$, which contain no points of $S$. Refer to these horizontal line segments directly above and below $p$ which do not intersect $S$ as $U_{p}$ and $L_{p}$ respectively. For each angle $x$, we define $R(x)$ to be the ray based at $q$ making an angle of $x$ with a horizontal line passing through $q$. Then there is an interval of angles, which we will call $I$, which is the set of all angles $x$ such that $R(x)$ intersects both $U_{p}$ and $L_{p}$.

Let $a$ and $b$ denote the left and right end points of $I$. Let $T$ be the trapezoid bounded by $R(a), R(b), L_{p}$ and $U_{p}$. We will refer to the "left side of $T$ " as the set of all $x \in T$ to the left of $p$. We refer to "the right side of $T$ " as the set of all angles $x \in T$ to the right of $p$. If there are arcs on both the left and right sides of $T$ which each contain points of $L_{p}$ and $U_{p}$ and do not intersect $S$, then the union of these arcs and $L_{p}$ and $U_{p}$ contain a simple closed curve which does not intersect $S$ and which bounds an open set which is contained entirely within $O$. This contradicts our assumption that no such open set exists, so we conclude that on either the right or left side of $T$ there is no arc which does not intersect $S$ and contains points of $U_{p}$ and $L_{p}$. Without loss of generality, we will assume it is the left hand side that contains no such arc.

We let $H$ be the set of angles $x \in I$ such that $R(x)$ contains points in the left side of $T$. For each angle $x \in H$, we let $x(1)$ and $x(2)$ denote the points of $R(x) \cap S$ which are contained in $T$ when two such points exist, and when two such points exist we let $x(1)$ refer to the higher of the two. If there is only one point on $R(x) \cap S$ in $T$, then refer to that point as $x(1)$. Note that there is always some point in $R(x) \cap S \cap T$ or else $R(x)$ contains a path from $L_{p}$ to $U_{p}$ which contains no points of $S$, contradicting our assumption. We will say that a point $x(i)$ (where $i$ could be either 1 or 2) is avoidable if for some $\epsilon>0$, for every $\delta>0$ there is a point $y \in H$ so that $d(x, y)<\delta$ and $d(x(i), y(j))>\epsilon$ for all $j$ such that $y(j)$ is defined.

Let $x \in H$ and let $R(x)$ contain an avoidable point $x(i)$. Then we may choose $\epsilon>0$ so that for every $\delta>0$ there is a point $y \in H$ so that $d(x, y)<\delta$ and $d(x(i), y(j))>\epsilon$ for all $j$ such that $y(j)$ is defined. Choose an open ball $N(x(i))$ about $x(i)$ of radius less than half the distance to $x(j)$ for any $j \neq i$, and also less than $\epsilon$, and also small enough so that $N(x(i))$ has a positive distance from the right side of $T$ and the line segments $L_{p}$ and $U_{p}$. There are horizontal line segments above and below $x(i)$ (not necessarily containing points directly above and below $x(i)$ ) which contain points of $R(x)$ in their interiors, and which are contained in $N(x(i))$ and do not intersect $S$. We refer to these horizontal line segments as $U_{x(i)}$ and $L_{x(i)}$. We can choose a point $y \in H$ which is close enough to $x$ that $R(y)$ intersects both $U_{x(i)}$ and $L_{x(i)}$ and so that $d(x(i), y(j))>\epsilon$ for all $j$ such that $y(j)$ is defined. Note that there are no points of $R(y) \cap S$ which are contained in $N(x(i))$. Hence, the arc constructed by taking the union of the line segments from the point $R(x) \cap L_{p}$ to $R(x) \cap L_{x(i)}$, from $R(x) \cap L_{x(i)}$ to $R(y) \cap L_{x(i)}$, from $R(y) \cap L_{x(i)}$ to $R(y) \cap U_{x(i)}$, from $R(y) \cap U_{x(i)}$ to $R(x) \cap U_{x(i)}$ and from $R(x) \cap U_{x(i)}$ to $R(x) \cap U_{p}$ is an arc contained entirely in the left side of $T$ which contains no points of $S$ not contained in $R(x) \cap S \cap T$ and does not contain, or avoids, the point $x(i)$. We refer to the path above as an arc avoiding $x(i)$. Notice that since $N(x(i))$ was chosen of radius less than half the distance $d(x(i), x(j))$, for $j \neq i$ if such an $x(j)$ is defined, if both $x(1)$ and $x(2)$ are avoidable, then if we take the union of an arc avoiding $x(1)$ and an arc avoiding $x(2)$ and remove those points of $R(x)$ which are not in both of these two arcs, then we obtain an arc from $L_{p}$ to $U_{p}$ which contains no points 
of $S$. We refer to an arc constructed in this way as an arc avoiding both $x(1)$ and $x(2)$. The existence of such an arc contradicts our assumption. Similarly, if $x(1)$ is avoidable and $x(2)$ is not defined, then an arc avoiding $x(1)$ contains no points of $S$ and this contradicts our assumption. Also, note that if for an unavoidable point $x(i)$ it is the case that for some $\epsilon>0$ for every $\delta>0$ there is some point $y \in H$ so that for any $j$ which is defined, either $d(x(i), y(j))>\epsilon$ or $y(j)$ is avoidable, then using an arc which avoids $y(j)$ and is contained in $R(y) \cup N(x(i))$ which intersects both $U_{x(i)}$ and $L_{x(i)}$ instead of $R(y)$ in the previous argument allows us to obtain a path from $L_{p}$ to $U_{p}$ which does not contain any points of $S$ not contained in $R(x) \cap S$ and does not contain, or avoids, the point $x(i)$. In this case we refer to the point $x(i)$ as a secondary avoidable point. Note that if $R(x) \cap T$ contains either only one point of $S$ which is a secondary avoidable point, or two points of $S$ which are secondary avoidable points, then in a similar manner as used to obtain an arc containing no points of $S$ contained in the left side of $T$ from $L_{p}$ to $U_{p}$ when the two points on $R(x)$ were avoidable, we may obtain such an arc when the two points on $R(x)$ are secondary avoidable points.

From the preceding argument we conclude that for every $x \in H$ there is some $i$, either 1 or 2 , such that $x_{i}$ is not avoidable. We will now show that for a dense set $D$ in $H$ there is exactly one $i$, either 1 or 2 , so that $x(i)$ is not avoidable. Suppose by way of contradiction that for some interval of points $K \subset H$, for each $x \in K$ both $x(1)$ and $x(2)$ are defined and neither is avoidable. Then let $x$ be a point in the interior of $K$. Let $\epsilon$ be one-half the distance from $x(1)$ to $x(2)$. We may choose $\delta>0$ such that if $d(x, y)<\delta$, then $y \in K$ and $d(x(1), y(j))<\epsilon$ for some $j$, either 1 or 2 , and $d(x(2), y(j))<\epsilon$ for some $j$, either 1 or 2 . Note that since there must be a $y(j)$ within $\epsilon$ of both $x(1)$ and $x(2)$, in the preceding sentence we can conclude that in the first instance $j=1$ and in the second instance $j=2$. We now define a function $g:[x-\delta / 2, x+\delta / 2] \rightarrow T$ by letting $g(z)=z(1)$. We claim that $g$ is a homeomorphism, so the image of $g$ is an arc, which cannot be a subset of $S$ because $S$ is a three-point set. It is clear that $g$ is one-to-one, onto, and that its inverse is continuous. We verify that $g$ is continuous. Let $\epsilon *>0$, and let $y$ be a point in the domain of $g$. We may choose $\delta *<\delta / 2$ such that if $d(y, z)<\delta *$, then $d(y(1), z(j))<\epsilon *$ for some $j$. Again, since in the previous sentence the distance from $x(1)$ to $z(j)$ is no more than half the distance from $x(1)$ to $x(2)$ and the distance from $x(2)$ to $z(j)$ is also no more than that distance for some $j$, we conclude that $j=1$ in the last sentence. Hence, we conclude that if $d(y, z)<\delta *$, then $d(g(y), g(z))<\epsilon *$, and so $g$ is a continuous function. Since there can be no arcs in a three-point set we conclude that there is a dense set $D$ so that for all $x \in D$, there is only one $i$, either 1 or 2 , so that $x(i)$ in not avoidable, since every interval in $H$ must contain such a point.

For each point $x \in D$ we let $x(k)$ denote the unavoidable point of $R(x)$. We define a function $f: D \rightarrow T$ by letting $f(x)=x(k)$, and we claim that the image of $f$ is a dense subset of an arc, and we wish to show further that this arc is a subset of $S$, thereby arriving at a contradiction to our original assumption that $S$ is zero dimensional. First, we show that the image of $D$ under $f$ is a dense subset of an arc. Since $f$ is bounded and $D$ is dense in $H$, the closure of $f(D)$ must contain a point of $R(x)$ for each $x \in H$. Suppose that $R(x)$ contains two points in the closure of $f(D)$, and call the distance between them $2 \epsilon$. Then it follows that both $x(1)$ and $x(2)$ are avoidable since for each such $x(i)$, there are points $y \in D$ arbitrarily 
close to $x$ so that $y(k)$ is a distance more than $\epsilon$ from $x(i)$. So, we define a function $h: H \rightarrow T$ to be the continuous extension of $f(x)$. The image of $h$ is an arc, and we will refer to this arc as $A$.

We will now show that $A \subset S$, which is a contradiction to the fact that $S$ cannot contain an arc. Suppose that $A$ is not a subset of $S$. Then let $h(x)$ be a point of $A$ not contained in $S$. Let $\epsilon$ be half the minimum distance from a point of $R(x) \cap S$ to $h(x)$. Since for every $\delta>0$ there is a point $y \in D$ such that $d(x, y)<\delta$ and $d(x(j), y(k))>\epsilon$ for all $j$, it follows that both $x(1)$ and $x(2)$ are secondary avoidable points, and hence there is an arc from $L_{p}$ to $U_{p}$ in the left side of $T$ which does not intersect $S$. This contradicts our assumption, and so it follows that there is an arc which is contained in $S$ which is impossible. We conclude that $S$ is zero dimensional.

Theorem 2.2. No three-point set can contain an arc.

Proof. Let $A$ be an arc, and suppose that $A$ is contained in a three-point set $S$. First we define "up" to be the direction perpendicular to the segment connecting the end points of $A$ so that other points of $A$ would be above the segment. Without loss of generality we will assume that no other points of $A$ are contained on the line connecting the end points of $A$ apart from the two end points (otherwise we replace $A$ by the arc above two consecutive points on that line and rename that $\operatorname{arc} A$ ). Since $A$ is compact there is a point of $A$ of maximum height, and we will refer to that point as $p$. There cannot be two such maximum height points, for if $q$ were a second point of maximum height, a horizontal line in this orientation, close enough to both $p$ and $q$, must necessarily intersect $A$ in four points since traversing $A$ in either direction along the arc from either local max must eventually contain points lower than the max (since at most three points can be on the horizontal line connecting $p$ and $q$ and all other points must be lower).

Similar to the above argument, traversing the arc in either direction from $p$, the height of points cannot decrease and then increase again. Let $p$ be the image of some point $x \in[0,1]$ under a homeomorphism $f:[0,1] \rightarrow A$, and suppose that for some points $y, z \in[0,1]$ such that $x<y<z<1$ it is true that $f(x)>f(z)>f(y)$ (where ">" indicates "higher than" in this case). Then we can pick a horizontal line of height between $h(z)$ and $h(y)$. This line must intersect the image of the segment from $y$ to $z$. It must also intersect the image of the segment from $x$ to $y$ and it must also intersect the image of the segment from $z$ to 1 . It must also intersect the segment from 0 to $x$ and hence the line must intersect the arc in four points, which is not possible. Hence, we conclude that the function $f$ is always decreasing in height in either direction from $p$.

The horizontal line containing $p$ must contain two other points of $S$, and we let $t$ be the nearest such point to $p$, and without loss of generality, we may assume $t$ is on the left side of $p$ and that $f(0)$ is to the left of $f(1)$. Let $C$ be the union of all lines passing through $t$ and any point $f(y)$ such that $y>x$. Note that each such line contains $t$ and two points of $A$ and hence cannot contain any additional points of $S$. Let $d$ be the distance from $t$ to $A$, and let $h(t)$ be the height of $t$ (the distance from $t$ to the segment connecting the end points of $A$ ). Let $h$ be a height chosen so that $h<h(t)$ and each horizontal line of height in the interval $[h, h(t)]$ intersects $C$ in a point which is a distance less than $d$ from $t$. Let $B$ be the horizontal band consisting of the union of all horizontal lines of height in the interval $[h, h(t)]$. We will define a map $g:[h, h(t)) \rightarrow B$ by stating that $g(z)$ is the 
unique point of intersection of the horizontal line of height $z$ with $S$ which is not a point of $A$. We wish to show that $g$ is continuous.

Suppose that $g$ is not continuous. Then for some point $z \in[h, h(t))$ for some positive number $\epsilon$, for each positive $\delta$ there is a point $w \in[h, h(t))$ so that $d(w, z)<$ $\delta$ and $d(g(w), g(z))>\epsilon$. There are no points in the image of $g$ on any line in the union $D$ of the lines through $g(z)$ and any point $f(y)$ such that $x<y<1$ since these lines already contain three-points of $S$. Choose a positive number $\delta$ so that every horizontal line of height within a distance of $\delta$ of $z$ intersects $D$ inside $N(g(z), \epsilon)$ on both the left and on the right of $g(z)$. This is possible since the height of $g(z)$ is between that of $p$ and that of $f(1)$. Then let $y$ be a point of $[h, h(t))$ a distance less than $\delta$ from $z$ so that $d(g(y), g(z))>\epsilon$. But then $g(y)$ cannot be within $\epsilon$ of $g(y)$ since all points of the horizontal line of height $y$ which are not within $\epsilon$ of $g(z)$ are contained in $D$, and $g(y)$ cannot be contained in $D$ because only points of $A$ and $g(z)$ are points of $S$ contained in $D$. This is a contradiction.

Hence, we conclude that $g$ is continuous. There is a third point $r$ on the line containing $p$ and $t$. By rotating this horizontal line slightly with $r$ as the axis we obtain a line which intersects $A$ in two places, and also must intersect the image of $g$, intersecting $S$ in four points which is a contradiction. More specifically, if $r$ is to the right of $p$, then a line through $r$ having a negative angle of smaller magnitude with the horizontal than a line through $r$ and $g(h)$ intersects $S$ in four points, and if $r$ is to the left of $t$ (only other possibility since $t$ was the closest point to $p$ in $S$ on that horizontal line), then a line through $r$ having a negative angle of smaller magnitude with the horizontal than the line through $r$ and $f(1)$ and the line through $r$ and $g(h)$ must intersect $S$ in four points. Hence, we conclude that $S$ cannot contain an arc.

\section{REFERENCES}

1. Jan J. Dijkstra, On n-point sets, Topology and Dynamics Conference, San Antonio, Texas, 2000.

2. Khalid Bouhjar, Jan J. Dijkstra, Jan Van Mill, Three point sets, Top. Appl., 112 (2001), 215-227. CMP 2001:10

3. B. Engelking, Dimension theory, PWN, Warszawa, 1978. MR 58:2753b

4. John Kulesza, A two point set must be zero dimensional, Proc. Amer. Math. Soc. 116 (1992), 551-553. MR 92m:54064

5. R. D. Mauldin, Problems in topology, Open Problems in Topology, J. Van Mill and G. M. Reed, eds., North-Holland, Amsterdam, 1990. MR 92c:54001

Department of Mathematics, Utah Valley State College, Orem, Utah 84058

E-mail address: davidfearnley@juno.com

Department of Mathematics, Brigham Young University, Provo, Utah 84602

Department of Mathematics, Brigham Young University, Provo, Utah 84602

E-mail address: jack@math.byu.edu 